\title{
Robotic endoscopic surgery in a porcine model of the infant neck
}

\author{
Russell A. Faust · Adrien J. Kant • Attila Lorincz • \\ Abbas Younes · Elizabeth Dawe · Michael D. Klein
}

Received: 27 October 2006/ Accepted: 28 November 2006/ Published online: 30 January 2007

(C) Springer London 2007

\begin{abstract}
Minimally invasive surgery is rapidly becoming the desired surgical standard, especially for pediatric patients. Infants and children are a particular technical challenge, however, because of the small size of target anatomical structures and the small surgical workspace. Computer-assisted robot-enhanced surgical telemanipulators may overcome these challenges by facilitating surgery in a small workspace. We studied the feasibility of performing robotic endoscopic neck surgery on a porcine model of the human infant neck. The study design was a prospective, feasibility pilot study of a small cohort for proof of concept and for a survival model. Sixteen non-survival piglets weighing $4.5-10 \mathrm{~kg}$ were used to develop the surgical approach and operative technique. Eight piglets aged 3-6 weeks old and weighing 4.0-9.1 kg underwent survival thyroidectomy by a cervical endoscopic approach using the Zeus surgical robot, which includes the Aesop endoscope holder and "Microwrist" microdissecting instruments. We succeeded in performing endoscopic robotic neck surgery on a piglet as small as $4 \mathrm{~kg}$, in an operative pocket as small as $2 \mathrm{~cm}^{3}$. Total incision length for all three ports was $\leq 23 \mathrm{~mm}$. There were no major complications, no major robotic instrument malfunctions or breakages, and no procedures required
\end{abstract}

Electronic supplementary material The online version of this article (doi: 10.1007/s11701-006-0007-5) contains supplementary material, which is available to authorized users.

R. A. Faust $(\bowtie) \cdot$ A. J. Kant · A. Lorincz .

A. Younes - E. Dawe · M. D. Klein

Children's Hospital of Michigan,

Wayne State University School of Medicine,

3901 Beaubien Street, Detroit, MI 48201, USA

e-mail: faustr@chi.osu.edu conversion to open surgery. These results support the feasibility of robotic endoscopic neck surgery on a neck the size of a human infant's.

Keywords Endoscopy/endoscopic $\cdot$ Infant $\cdot$ Neck dissection · Pediatric - Robot - Robotic surgery · Thyroidectomy $\cdot$ Aesop $\cdot$ Hermes $\cdot$ Zeus

\section{Introduction}

As minimally invasive surgery emerges as the desired standard, endoscopic methods have developed to meet this objective. In some surgical disciplines, and for some surgical procedures, endoscopic methods are the preferred technique, for example in laparoscopy and thoracoscopy [1-5]. Endoscopic applications in the field of otolaryngology (head and neck surgery) have made significant advances in endoscopic sinus surgery and in endoscopic craniofacial surgery for both traumatic and cosmetic reconstruction [6-8]. More recently, pioneers of endoscopic surgery have begun to adapt techniques that were originally developed for body cavities (e.g. laparoscopy and pelviscopy) to operate in the axillae and other anatomic regions where cavities are not found [9-12]. In the past ten years innovative surgeons have begun to test the limits of endoscopic techniques for parathyroidectomy and thyroidectomy in humans [13-18]. Others have begun to model more extensive endoscopic neck procedures in animal models [19-22]. The transition to endoscopic surgery challenges the surgeon with fewer degrees of freedom, less tactile ("haptic") feedback, amplification of small movements into large translated movements because of the 
length of endoscopic instruments, and body postures for the surgeon that are often ergonomically unsatisfactory. All of these challenges are amplified with the pediatric patient. The advent of computer-assisted surgical robots improves on most of these challenges. Pediatric surgeons have been particularly interested in endoscopic and robot-enhanced surgery, because pediatric surgeons apply minimally invasive techniques to a broader range of procedures than do adult surgeons. The objectives of minimally invasive techniques, including minimizing postoperative pain and optimizing cosmesis, are paramount in the pediatric patient. Thus, pediatric surgeons are extending these techniques to infant, newborn, and even fetal procedures [23-30]. Endoscopic surgery on the infant patient is, however, characterized by particular challenges in addition to those present in the adult analog:

- body cavities are smaller, with the result that the effects of tremor and imprecision are magnified;

- endoscopic "exposure," or visibility, is reduced;

- organs and tissues are smaller and more delicate, and thus more susceptible to injury; and, finally

- the closer confines of the anatomy necessitate close proximity of camera and instruments, with increased potential for collision.

As a result, although minimally invasive endoscopic or robotic surgery may have benefits that are most desired in the pediatric patient population, it is precisely this population that poses the greatest challenge to applying these techniques [27]. Inspired by pioneering work in endoscopic neck surgery [21, $22,31,32]$, and by robotic endoscopic surgery in ever-smaller spaces [28, 30,33], we sought to determine whether robotic surgery could be successfully performed on a survival animal model with a neck the size of a human infant. Previous reports of endoscopic neck surgery that have used a porcine model of the adult neck have employed pigs ranging in size from 20 to $60 \mathrm{~kg}$ [21, 22, 34, 35]. In addition to the challenges described above, further limitations to performing robotic endoscopic surgery on smaller models have included the size limits, the types of instrument currently available, and lack of optimum animal models for neck surgery. On the basis of recent reports of successful endoscopic neck procedures on the porcine model we decided to determine the feasibility of performing robotic endoscopic neck surgery on much smaller pigs. Our experience developing a technique for thyroidectomy as a test procedure for "proof of concept" on piglets ranging in size from 4 to $9 \mathrm{~kg}$ is reviewed below.

\section{Methods}

Animals were cared for humanely according to Institutional Animal Care and Use Committee Guidelines at Wayne State University School of Medicine. The committee approved all procedures, and animals were under the constant care of a veterinarian. A total of 24 live animal dissections were performed.

\section{Non-survival phase I}

Sixteen live, non-survival piglets weighing between 4.5 and $10 \mathrm{~kg}$ were operated on to gain familiarity with porcine neck anatomy, and to conceptualize and develop the robotic endoscopic technique, including refinement of robot arms and camera placement, trocars placement, balloon and blunt dissection technique, and insufflation technique in the neck. Most non-survival animals underwent other surgical procedures either before or after robotic endoscopic neck surgery, primarily intra-abdominal robotic endoscopic procedures. Open neck dissection was undertaken to examine anatomic planes and port positions only when surgery could not be completed using the surgical robot. Notes on operative times were kept during the latter half of Phase I development.

\section{Survival phase II}

Eight survival animals, weighing between 4.0 and $9.1 \mathrm{~kg}$ underwent robotic endoscopic thyroidectomy. Survival animals underwent neck surgery only. A surgical log was kept, and procedures were photographed and recorded, for qualitative and quantitative recording of the experience.

\section{Surgical procedure}

Animals were fasted for $16 \mathrm{~h}$ before surgery, although water was provided. Anesthesia was induced with ketamine (22 $\left.\mathrm{mg} \mathrm{kg}^{-1} \mathrm{IM}\right)$ and acepromazine $(1.1 \mathrm{mg}$ $\left.\mathrm{kg}^{-1} \mathrm{IM}\right)$, and buprenorphine $\left(0.01 \mathrm{mg} \mathrm{kg}^{-1} \mathrm{IM}\right)$ for preemptive analgesia. The animals were intubated and isoflurane $(2-3 \%)$ was administered for general anesthesia. Respiration was supported with a mechanical ventilator. After first optimizing procedures and techniques during Phase I, procedures on infant piglets were performed in a sterile operating room environment (Phase II). After induction of anesthesia the neck was shaved and prepared and draped in a sterile manner, with the piglet in the supine position. The Zeus Surgical Robot (Computer Motion, CMI, Goleta, 
CA, USA) with two instrument arms and the Aesop video arm (controlled by means of the Hermes voiceactuated interface from Computer Motion) were used. The robotic system was set up before skin preparation and then draped into the sterile field with the animal. It consists of several units (Fig. 1): the robotics unit, with surgeon hand controls, is positioned behind the seated surgeon. The system provides the surgeon with voice control of the Aesop camera arm and voice control of most of the electronic devices in the OR, through a headset microphone. A high-definition monitor is placed low in front of the surgeon, providing the surgeon with a view of the operating table, patient, robot, and surgical assistant. The three robotic arms are mounted separately on the OR table rails. Although many instruments were experimented with during development of techniques in Phase I, we decided to use the smallest smooth-jawed grasping instruments
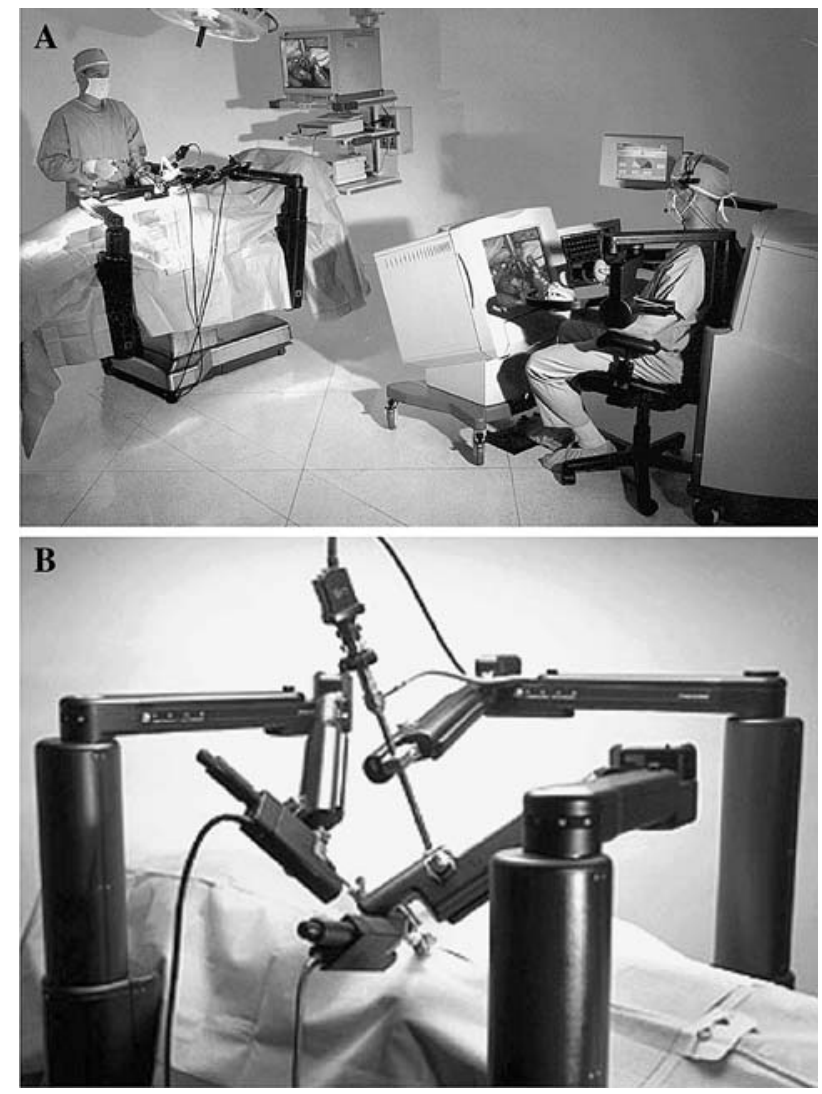

Fig. 1 Zeus surgical robot. a Zeus surgical telemanipulator system. Surgeon sits at control console at right, with control unit behind, and three monitors in front. The large monitor furnishes a high-resolution two-dimensional endoscopic view of the surgical workspace; the next monitor to the right shows Hermes voice-commands for the Aesop camera holder; the third monitor shows motion-scaling and other details of robotic control. b Robotic instrument arms and Aesop endoscope arm (not sterile-draped) at the operating table available at that time, in both robot arms, for dissection of tissue of survival animals. These were the Zeus micro-needle holders, with a jaw width of $1 \mathrm{~mm}$ and a "hand" length of $7 \mathrm{~mm}$ (Fig. 2), For Phase II survival piglets, three ports (Ethicon, Cincinnati, OH, USA) were used (Fig. 3). An 11-mm transverse cervical incision was created in the midline between the mandibular angles, the platysma was divided sharply, and an 11-mm non-bladed trocar was introduced into the subplatysmal plane toward the sternal notch in the midline. Dissection in the sub-platysmal plane was performed either bluntly, using the spreading technique with "Kelly" forceps, or by insertion of a $1,000 \mathrm{~cm}^{3}$ "pre-peritoneal dissection (hernia) balloon" (AutoSuture OMS-PDB 1,000; US Surgical, Norwalk, CT, USA), with an attempt made to inflate the balloon to approximately $300 \mathrm{~cm}^{3}$. In extremely small animals the balloon could not be inflated and merely provided a means of blunt dissection. This dissection facilitated instrument port placement and developed the central operative pocket, which remained a potential space until insufflated. After dissection an $11 \mathrm{~mm}$ trocar was reinserted through the incision and was used as the port for the $0^{\circ} 10 \mathrm{~mm}$ endoscope and camera during the procedure, held and controlled by Aesop. The same port was used for retrieval of the resected thyroid specimen at the end of the procedure. Insufflation with $\mathrm{CO}_{2}$ was initiated, and the flow was varied spontaneously to maintain a constant pocket pressure of $4 \mathrm{mmHg}$. This inflated the operative pocket to several square centimeters, as measured by direct comparison with the instruments. During the procedure the animal's body temperature, heart rate, electrocardiogram, and oxygen saturation were monitored. Two 5-mm trocars were inserted laterally under endoscopic visu-

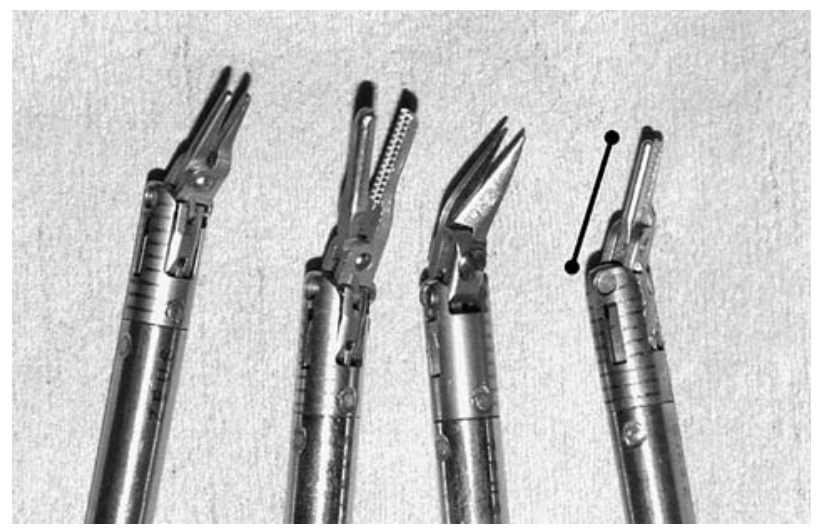

Fig. 2 Zeus "MicroWrist" robotic instruments. This is a small sample of available Zeus robotic instruments. This study used the micro-needle holder shown at the far right, with a jaw length of $7 \mathrm{~mm}$, and overall "hand" length of $11 \mathrm{~mm}$ (line) 


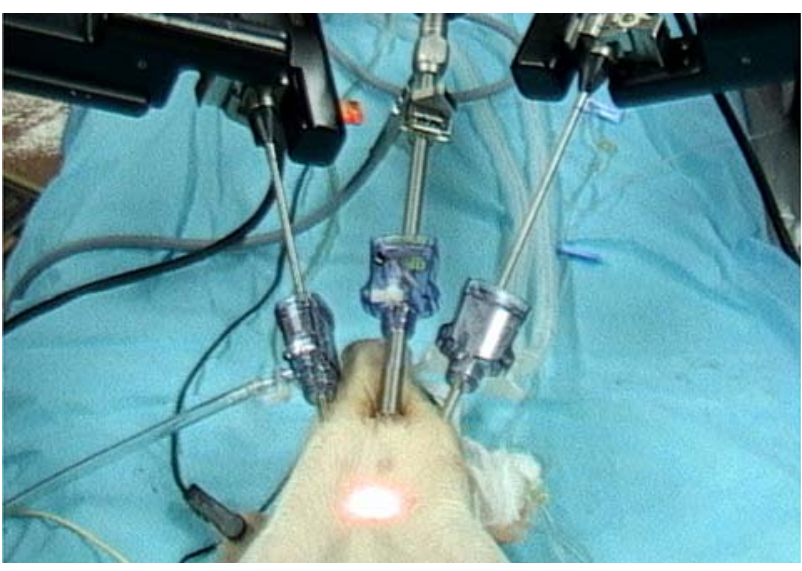

Fig. 3 Cervical approach for robotic endoscopic thyroidectomy. Surgical set-up with ports in place, and superior cervical endoscopic approach for robotic instruments and endoscope, with insufflation attached to instrument port at right neck; nonsurvival series. Piglet in supine position, with head toward top of figure

alization, entering just inferior to the mandibular angle on either side through stab-incisions with a no. 11 blade (Fig. 3). These operative ports introduced Zeus instruments (Figs. 2,3) to the operative pocket. After dissection and isolation of the thyroid gland, one of the $5 \mathrm{~mm}$ instruments was removed; a $0^{\circ} 5 \mathrm{~mm}$ endoscope was inserted through its port, and the specimen was retrieved through the $11 \mathrm{~mm}$ trocar using a simple endoscopic grasper under endoscopic visualization. After removal of the thyroid specimen the $10 \mathrm{~mm}$ endoscope was returned to Aesop and used to visually inspect the operative cavity for injuries or hemorrhage. Insufflation was stopped, instruments were removed, and incisions sutured. Postoperative diet was ad lib. The Phase II survival piglets were killed one week postoperation. Postoperative medication included an analgesic, buprenorphine ( $0.05 \mathrm{mg} \mathrm{kg}^{-1} \mathrm{IM}$ prn), and topical antibiotic ointment applied to the surgical incisions for two days postoperatively. Thyroid was confirmed histologically; post-mortem dissections were performed to inspect for evidence of nerve or vessel injury. Data collected included pre and postoperative weight and operative times. Anesthesia time was measured from induction to incision closure. Robotic setup time included the entire sterile prep, draping of the pig and robot, readying the arms and moving them into position. Port placement included the initial balloon dissection and insertion of both instrument ports when the camera was in place. Operating time referred to the period of robotic surgery from when instruments were introduced until the thyroid was dissected free. Extraction time and time to close incisions were also recorded.

\section{Results}

Training and development

Training for computer-assisted robot-enhanced surgery requires demonstration of improving times to perform a number of standard tasks on inanimate objects, including forehand and backhand suturing and knottying with both right and left hands. This phase of training exceeded $50 \mathrm{~h}$ and was followed by a variety of surgical tasks on non-survival animals. Much of the development effort of Phase I was spent optimizing robot arm placement, instrument port placement, and camera placement, to optimize surgical access to the intracorporeal surgical workspace of the central neck, and to minimize proximity conflicts of the extracorporeal robotic apparatus. Overall, time logged on the Zeus surgical robot for both Phases I and II of this study was greater than $120 \mathrm{~h}$.

\section{Non-survival phase I}

The entire surgical procedure was performed through incisions with a total length of $\leq 23-11 \mathrm{~mm}$ for the camera port, and 5-6 $\mathrm{mm}$ for each of the two instrument ports. It is worthy of note that the two instrument ports may be placed in the axillae to minimize the length of incision in the neck for optimum cosmesis. We experimented with this axillary approach during the Phase I non-survival series but found that, although the pig lacks a clavicle thereby simplifying access to the neck from the axillae, the sternal keel in pigs of extremely small size interferes with thyroid access by this approach. This resulted in prohibitively longer operative times and the inability to complete the procedure without converting to the superior cervical approach. The axillary approach was therefore abandoned in our smaller, survival series of infant piglets. This result is similar to that recently reported by Terris' group in a prospective evaluation of various approaches to thyroidectomy in the porcine model [32]. The porcine model is therefore limited to demonstrating feasibility, and for human patients the axillary approach would be considered for cosmetic reasons. Two intra-operative deaths occurred early in our Phase I development-one apparently because of insufflation pressures $(12 \mathrm{mmHg})$ that interfered with cervical venous flow [20, 34] and another which occurred during mediastinal dissection that resulted in pressure against the aortic arch by one of the robotic instruments. Postmortem examinations of these animals revealed no other injuries. All subsequent procedures were performed at an insufflation pressure of $4 \mathrm{mmHg}[21,22$, 
34] All but four thyroid glands were successfully excised by use of the robotic endoscopic surgical technique on the 16 non-survival piglets. An early cohort of seven piglets enabled us to become familiar with the relevant anatomy, determine appropriate positions for the pig and robot on the table and for camera and port placement, trial the dissection balloons, refine dissection techniques, and compare inferior cervical and axillary, and superior cervical approaches. A later group of nine non-survival piglets was used to further refine and standardize operative technique. Although we did not record operative times of our first seven non-survival operations, the times decreased with experience over the last nine animals for which times were recorded. On the basis of videotapes and written logs for these procedures, operative times decreased from the longest of $80 \mathrm{~min}$ to the shortest of $10 \mathrm{~min}$ during Phase I. An initial inferior approach was unsuccessful despite trying a variety of instrument-port placements. After switching to the superior approach we encountered difficulties in three cases that prevented completion of thyroidectomy. The necks of these three non-survival piglets during Phase I were dissected openly immediately after surgery to clarify anatomic planes and identify areas for improvement. In two of these cases port placement and/or dissection was too superficial, leading to problems with perception of and access to the central neck compartment. These dissections enabled us to confirm the anatomy and adjust camera and instrument placements for optimum visualization and effective dissection in subsequent procedures.

\section{Survival phase II}

In the survival cohort of eight piglets no conversions to open surgery were necessary. There were no intraoperative complications in the survival series. Overall, the robotic system was precise and thyroidectomy was readily performed in infant piglets as small as $4.0 \mathrm{~kg}$ (Fig. 4). Total duration of the sterile operative procedure in survival animals ranged from 83 to $119 \mathrm{~min}$ (Table 1). As is evident from Table 1, there was no appreciable trend toward improving operative times during this survival series; this probably reflects the extensive training that was achieved before and during the non-survival Phase I development period. Unlike the human thyroid, the porcine thyroid lacks a significant neuro-vascular association; analogous to the human thyroid, it is invested in a thin capsule, lies deep to the midline strap musculature, within adipose tissue and fascia, against the anterior tracheal wall. Despite the lack of need for vascular ligation, we routinely surveyed the surgical cavity for hemorrhage after removal of the thyroid (Fig. 4). We also performed one-week survival follow up to confirm the safety and tolerance of the procedure in the piglets. All survival animals resumed their regular diet within hours of surgery, and continued water consumption, food consumption, and weight gain (Table 1) in parallel with a non-operated cohort of age-matched infant piglets.

\section{Complications}

For two of the survival animals cervical subcutaneous emphysema was apparent immediately after completion of the procedure; this dissipated over the following $24 \mathrm{~h}$ without sequelae. There were three

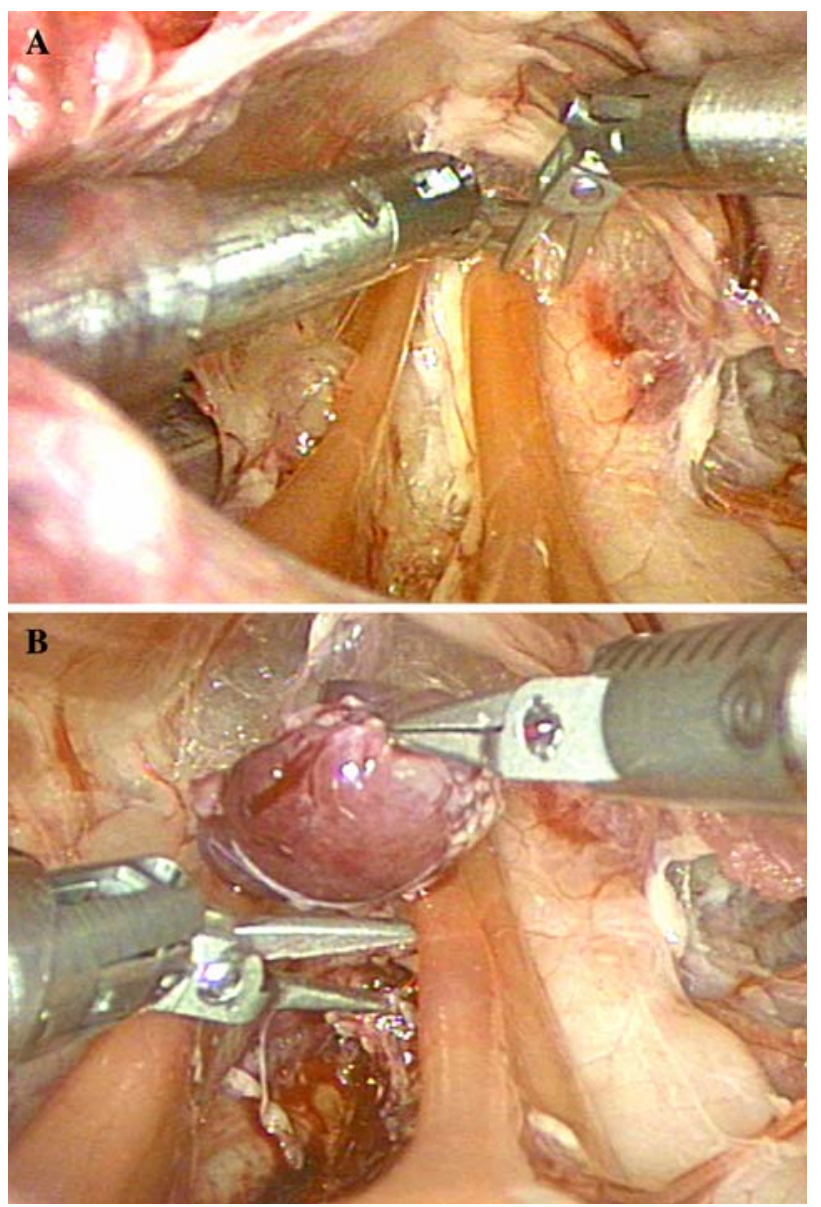

Fig. 4 Endoscopic view during robotic endoscopic thyroidectomy. a View immediately after balloon dissection, insertion of robotic instruments, and insufflation. Note long, narrow, anterior strap muscles, beneath which lies the thyroid. b View at end of dissection with the thyroid free and in the grasp of the right-hand instrument. For size reference: instrument diameter is $5 \mathrm{~mm}$; "jaw" length is $7 \mathrm{~mm}$ 
Table 1 Robotic endoscopic thyroidectomy in piglet model of the infant neck, with operative times

\begin{tabular}{|c|c|c|c|c|c|c|c|c|c|c|}
\hline Piglet no. & 1 & 2 & 3 & 4 & 5 & 6 & 7 & 8 & Mean & SD \\
\hline Weight at surgery (kg) & 9.1 & 7.0 & 4.2 & 5.8 & 4.9 & 5.2 & 4.0 & 4.5 & 5.6 & \pm 1.7 \\
\hline Weight 1 week post-op $(\mathrm{kg})$ & 13.3 & 9.1 & 5.0 & 6.3 & 5.4 & 7.0 & 6.0 & 5.9 & 7.3 & \pm 2.7 \\
\hline Anesthesia time (min) & 111 & 119 & 83 & 107 & 92 & 104 & 103 & 89 & 101 & \pm 12.1 \\
\hline Robotic setup time (min) & 35 & 32 & 34 & 37 & 31 & 39 & 37 & 35 & 35 & \pm 2.7 \\
\hline Port placement (min) & 12 & 15 & 11 & 10 & 10 & 11 & 14 & 21 & 13 & \pm 3.7 \\
\hline Operating time (min) & 23 & 40 & 9 & 31 & 10 & 22 & 15 & 8 & 20 & \pm 11.5 \\
\hline Extract thyroid (min) & 3 & 3 & 2 & 5 & 3 & 2 & 6 & 3 & 3 & \pm 1.4 \\
\hline Close incisions (min) & 20 & 13 & 14 & 11 & 12 & 14 & 19 & 10 & 14 & \pm 3.6 \\
\hline
\end{tabular}

minor complications in survival animals. One animal (piglet no. 3 of 8 ) developed a superficial wound abscess with erythema and purulence at the suture line of the left neck instrument port. This animal did not develop fevers, did not appear bothered by the site, and did not alter its diet, and thus the decision was made not to administer systemic antibiotics. The wound was treated by application of topical antibiotic ointment, and local swelling improved over the next two days. For all subsequent piglets in this series, topical antibiotic ointment was applied to the three suture lines after completion of surgery, and twice a day thereafter. One animal (piglet no. 7 of 8 ) developed a midline seroma pocket at the area of the suprasternal notch, without erythema or apparent discomfort. This animal did not develop fevers, did not alter dietary intake, and did not require systemic antibiotics. After review of the intra-operative video for this animal it became evident that the initial subplatysmal trocar dissection for the endoscope was more superficial, and the resulting tunnel longer, than was usual. It is uncertain whether this contributed to formation of the seroma. Finally, one animal (piglet no. 8 of 8) developed necrosis of a portion of thymus at the left neck instrument site, resulting in a small, watery, non-purulent fluid collection in the left neck, noted on necropsy. The pig thymus is situated longitudinally along either side of the trachea, and the introduction of the left instrument trocar had traversed thymic tissue during placement, resulting in this minor complication. There were no sequelae from this injury during the one-week survival period. There were no major complications, and no animal had to be surgically re-explored or euthanized as a result of complications. These three minor complications did not interfere with postoperative fluid or food consumption or growth until sacrifice at one week, and might have been expected to resolve had they survived longer.

\section{Discussion}

Minimally invasive techniques continue to revolutionize surgery. Endoscopic techniques have become the standard surgical approach to many disease processes. Robotic surgery has the potential to further extend minimally invasive surgical techniques into ever smaller cavities, and into body spaces where there are no natural cavities, for example the retroperitoneum or the neck fascial planes. In this study we succeeded in performing robotic endoscopic neck surgery on an infant piglet model as small as $4 \mathrm{~kg}$, in an operative pocket volume as small as $2 \mathrm{~cm}^{3}$. There were no major complications and no robotic instrument malfunctions or breakages requiring conversion to open surgery. As far as we are aware this is the first report of a study of the feasibility of endoscopic neck surgery in a model of this small size, and the first report of robotic endoscopic surgery in a survival model of the infant neck. In our experience, the most beneficial enhancements over traditional non-articulated endoscopic instruments offered by robotic technology include tremor-filtration, magnification, motion scaling from large operator movements down to microscopic movements at the level of robotic surgical instruments, and, most significantly, addition of the wrist to the endoscopic instruments. All surgeons have some tremor in the motion of their hands. This can become particularly problematic under magnification, at the end of long endoscopic instruments and during fine maneuvers in very small operative fields. The computer interface of surgical robots filters these minuscule motions when translating the surgeon's hand movements at the controls to the instrument tips, effectively eliminating tremors. The robotic interface can also translate extensive movements by the surgeon's hands at the controls to slower, smaller movements at the level of the robotic instruments_-"motion scaling". This enhancement enables such intuitive procedures as suturing and knot tying, 
especially within a very small operative cavity during robotic endoscopic surgery. Although our thyroidectomy porcine model did not require suturing or ligation, even simple tissue dissection was greatly facilitated by the articulated instruments. At the time this work was performed the Zeus surgical robotic system had several advantages over alternative systems available during the development of the work [27]. The other FDA-approved surgical robot available was the da Vinci, manufactured by Intuitive Surgical (Sunnyvale, CA, USA). It also holds minimally invasive instruments but differs from Zeus in several important features. The da Vinci wrist is very fluid and responsive, and boasts a range of at least $90^{\circ}$ in two planes. It required $8-\mathrm{mm}$ diameter instruments that had a minimum of $18.5 \mathrm{~mm}$ "hand" length, however; 5-mm diameter instruments are now available, but they require an even greater turning radius for the hand. This was the most limiting feature of the da Vinci for our purpose of robotic endoscopic surgery in the infant neck, where the workspace was often limited to a cube with a side of $20-25 \mathrm{~mm}$. Zeus instruments had a minimum jaw length of $7 \mathrm{~mm}$, resulting in a "hand" size of $11 \mathrm{~mm}$ (Fig. 2), but they moved through only $60^{\circ}$, and are limited to only a single wrist plane of motion. Because of our intent to model the human infant neck, we assumed an upper limit to neck width of $80 \mathrm{~mm}$. Based on the "hand" and wrist specifications of the two robotic systems, it became clear that the da Vinci has a lower limit of distance between instrument ports of $100 \mathrm{~mm}$; Zeus had a lower interport limit of less than $30 \mathrm{~mm}$ [27]. Thus, the da Vinci was not suited to our model. The lower limits for endoscope-to-instrument-tip for the da Vinci $(38 \mathrm{~mm})$ were also outside our theoretical limits for workspace dimensions (20-25 mm) whereas the Zeus (14 mm) was within these limits [27]. Since the acquisition of Computer Motion by Intuitive Surgical in 2003, support for the Zeus robotic system has been discontinued. It is, however, worthy of note that Intuitive Surgical has stated a commitment to further develop the da Vinci system for procedures in smaller surgical fields.

The total length of incision for all three ports in our survival animals was $23 \mathrm{~mm}$, which is similar to reports for endoscopic neck surgery models in the literature $[21,22,35]$. This total may, however, be further reduced by using a $5 \mathrm{~mm}$ endoscope, which Aesop does accommodate, and the da Vinci system has a 3D endoscope with a diameter of $8 \mathrm{~mm}$. This would bring the total incision length for the procedure to $20 \mathrm{~mm}$ or less. The central endoscope incision was also the port used for balloon dissection, however, and currently available dissection balloons require a $10-11 \mathrm{~mm}$ incision. We found that this could be avoided simply by using blunt dissection through a smaller incision. Using an axillary approach for the instrument ports also has the potential to further reduce the length of incision in the neck, to further optimize cosmesis. In adult-size pigs others have found this approach to be feasible, albeit slower and more difficult [32]. In Phase I with much smaller piglets we found the axillary approach to be prohibitive using the surgical robot, because of the thyroid's location in relation to the sternal keel in the smaller animals. The axillary approach has been successfully used for human non-robotic endoscopic thyroidectomy [36], however, and should be considered for its potential cosmetic benefits as an approach for human robotic endoscopic neck surgery.

Despite working with the smaller and more agile Zeus system, and despite optimization and standardization of robotic arm placement and instrument angles, painstakingly established during Phase I, we often experienced extra corporeal instrument-arm-to-camera proximity conflicts and instrument-instrument-arm proximity conflicts. This never resulted in termination or significant delay of the procedure; it was merely an annoyance, resulting in minor limitation of range of motion of one of the instruments. For infant piglets in the size range 4-6 kg, with inter-port distances of 4-6 cm, we were, however, routinely working at the limits of the available technology for robotic endoscopic neck surgery. Since discontinuation of support for the Zeus surgical robotic system, it remains to be established what proximity conflicts the larger remaining available robotic system, da Vinci, might have in the confines of similar small models. It also remains to be determined whether these potential proximity conflicts impose a real limit on robotic endoscopic neck surgery in smaller animal models, or smaller human infant patients, for which ports may be differently located because of anatomic disparities. Current abilities would benefit greatly from development of software to guide port placement with the goal of minimizing proximity conflicts.

As the safety and reliability of surgical robotics have become established, it is evident they may have some advantages, although they remain limited by current constraints. Further development and miniaturization may resolve some current limitations that we encountered. We have not introduced the issue of cost, but this will weigh heavily in the trend toward minimally invasive robotic surgery. With a single surgical robotic system costing over US \$1 million, significantly lower morbidity and quicker recovery will need to be demonstrated to justify use of these systems. For some 
surgical procedures it seems that robotic surgery has met that test, and is the evolving standard surgical approach [28, 37]. Whether there will be sufficient justification for application of computer-assisted surgical robots to endoscopic neck procedures remains to be determined. Pioneering work by other groups will help determine the value of robotic endoscopic surgery in the head and neck [38-40]. The infant porcine neck model described here establishes the feasibility of performing computer-assisted robot-enhanced endoscopic surgery in a neck the size of a human infant's. For the pediatric surgeon, robotic endoscopic surgery can help optimize the advantages of minimally-invasive techniques. It is uncertain at this time which surgical procedures may benefit most from the robotic endoscopic technique in human infants or children. We expect, however, that, as was found for early laparoscopic methods, clinical applications may evolve from this initial demonstration of ability. It is our hope that the infant porcine neck model described here will provide inspiration and incentive for further development of the tools and techniques for application of robotic technology to pediatric head and neck surgery.

Acknowledgments A portion of this study was presented as a Candidate's Thesis (R.A. Faust) before the annual meeting of the Triological Society, Boca Raton, FL, in May 2005.

\section{References}

1. Cuschieri A, Dubois F, Mouiel J, et al (1991) The European experience with laparoscopic cholecystectomy. Am J Surg 161:385-387

2. Monson JR, Hill AD, Darzi A (1995) Laparascopic colonic surgery. Br J Surg 82:150-157

3. Laine S, Rantala A, Gullischsen R, Ovaska J (1997) Laparascopic vs. conventional Nissen fundoplication: a prospective randomized study. Surg Endosc 11:441-444

4. Hansen JB, Smithers BM, Schache D, Wass DR, Miler BJ, Menzies BL (1996) Laparoscopic vs. open appendectomy. World J Surg 20:17-20

5. Nakajima J, Takamoto S, Tanaka M, Takeuchi E, Murakawa T, Fukami T (2001) Thoracoscopic surgery and conventional open thoracotomy in metastatic lung cancer. Surg Endosc 15:849-853

6. Kennedy DW (1985) Functional endoscopic sinus surgery: technique. Arch Otolarygol 111:643-649

7. Papay FA, Stein JM, Dietz JR, Luciano M, Morales L Jr, Zins J (1997) Endoscopic approach for benign tumor ablation of the forehead and brow. J Craniofac Surg 8:176-180

8. Alford EL (2001) Endoscopic browlift is the superior method for rejuvenation of the forehead and brow. Arch Otolaryngol Head Neck Surg 127:90-91

9. Fridlander L, Sundin J (1993) Minimally invasive harvesting of latissimus dorsi. Plast Reconstr Surg 94:881-884

10. Salvat J, Knopf JF, Ayoubi JM, et al (1996) Endoscopic exploration and lymph node sampling of the axilla: preliminary findings of a randomized pilot study comparing clinical and anatomo-pathologic results of endoscopic axillary lymph node sampling with traditional surgical treatment. Eur J Obstet Gynecol Reprod Biol 70:165-173

11. Ho LCY (1993) Endoscopic assisted transaxillary augmentation mammaplasty. Br J Plast Surg 46:332-336

12. Jugenheimer M, Jugenheimer T (1992) Endoscopic subfascial sectioning of incompetent perforating veins in treatment of primary varicosis. World J Surg 16:971-975

13. Gagner M (1996) Endoscopic subtotal parathyroidectomy in patients with primary hyperparathyroidism. Br J Surg 83:875

14. Miccoli P, Neninelli C, Vignali E, et al (1998) Endoscopic parathyroidectomy: report of an initial experience. Surgery 124:1077-1079

15. Huscher CSG, Chiodini S, Napolitano C, Recher A (1997) Endoscopic right thyroid lobectomy. Surg Endosc 11:877

16. Yeung GHC (1998) Endoscopic surgery of the neck. Surg Laparosc Endosc 8:227-232

17. Bellantone R, Lombardi CP, Raffaelii M, Rubino F, Boscherini M, Perilli W (1999) Minimally invasive, totally gasless video-assisted thyroid lobectomy. Am J Surg 177:342343

18. Shimizu K, Akira S, Jasmi AY, Kitamura Y, Kitagawa W, Akasu H, Tanaka S (1999) Video-assisted neck surgery: endoscopic resection of thyroid tumors with a very minimal neck wound. J Am Coll Surg 188:697-703

19. Brunt LM, Jones DB, Wu JS, Quasebarth MA, Meininger T, Soper NJ (1997) Experimental development of an endoscopic approach to neck exploration and parathyroidectomy. Surgery 122:893-901

20. Carreno OJ, Wilson WR, Nootheti PK (1999) Exploring endoscopic neck surgery in a porcine model. Laryngoscope 109:236-240

21. Monfared A, Saenz Y, Terris DJ (2002) Endoscopic resection of the submandibular gland in a porcine model. Laryngoscope 112:1089-1093

22. Terris DJ, Monfared A, Thomas A, Kambham N, Saenz Y (2003) Endoscopic selective neck dissection in a porcine model. Arch Otolaryngol Head Neck Surg 129:613-617

23. Heller K, Gutt C, Schaeff B, Beyer PA, markus B (2002) Use of the robotic system da vinci for laparoscopic repair of gastro-oesophageal reflux in children. Eur J Pediatr Surg 12:239-242

24. Le Bret E, Papdatos S, Folliguet T, et al (2002) Interruption of patent ductus arteriosus in children: robotically assisted versus videothoracoscopic surgery. J Thorac Cardiovasc Surg 123:973-976

25. Hollands CM, Dixey LN (2002) Robotic-assisted esophagoesophagostomy. J Pediatr Surg 37:983-985

26. Hollands CM, Dixey LN (2002) Applications of robotic surgery in pediatric patients. Surg Laparosc Endosc Percutan Tech 12:71-76

27. Drasin T, Gracia C, Atkinson J (2003) Pediatric applications of robotic surgery. Pediatr Endosurg Innov Tech 7:377-384

28. Knight CG, Lorincz A, Gidell KM, Lelli J, Klein MD, Langenburg SE (2004) Computer-assisted robot-enhanced laparoscopic fundoplication in children. J Pediatr Surg 39:864-866

29. Aaronson OS, Tulipan NB, Cywes R, et al (2002) Robotassisted endoscopic intrauterine myelomeningocele repair: a feasibility study. Pediatr Neurosurg 36:85-89

30. Knight CG, Lorincz A, Johnson A, Gidell KM, Rabah R, Klein MD, Langenburg SE (2004) Robot-enhanced fetoscopic surgery. J Pediatr Surg 39:1463-1465

31. Terris DJ, Haus BM, Gourin CG (2004) Endoscopic neck surgery: resection of the submandibular gland in a cadaver model. Laryngoscope 114:407-410 
32. Terris DJ, Haus BM, Nettar K, Ciecko S, Gourin CG (2004) Prospective evaluation of endoscopic approaches to the thyroid compartment. Laryngoscope 114:1377-1382

33. Lorincz A, Knight CG, Langenburg SE, Rabah R, Gidell K, Dawe E, Grant S, Klein MD (2004) Robot-assisted minimally invasive Kasai portoenterostomy. A survival porcine study. Surg Endosc 18:1136-1139

34. Rubino F, Pamoukian VN, Zhu JF, Deutsch H, Inabnet WB, Gagner M (2000) Endoscopic endocrine neck surgery with carbon dioxide insufflation: the effect on intracranial pressure in a large animal model. Surgery 128:1035-1042

35. Dulgerov P, Vaezi AE, Belenger J, et al (2000) Endoscopic neck dissection in an animal model: comparison of nodal yield with open-neck dissection. Arch Otolaryngol Head Neck Surg 126:417-420
36. Ikeda Y, Takami H, Sasaki Y, Kan S, Niimi M (2000) Endoscopic resection of thyroid tumors by the axillary approach. J Cardiovasc Surg (Torino) 41:791-792

37. Patel VR, Tully AS, Holmes R, Lindsay J (2005) Robotic radical prostatectomy in the community setting - the learning curve and beyond: initial 200 cases. J Urol 269-272

38. Haus GM, Kambham N, Le D, et al (2003) Surgical robotic applications in otolaryngology. Laryngoscope 113:1139-1144

39. Hockstein NG, Nolan PJ, O'Malley BW, Woo YJ (2005) Robot-assisted pharyngeal and laryngeal microsurgery: results of robotic cadaver dissections. Laryngoscope 115:10031008

40. O'Malley BW, Weinstein GS, Snyder W, Hockstein NG (2006) Transoral robotic surgery (TORS) for base of tongue neoplasms. Laryngoscope 116:1465-1472 